\title{
Performance analysis of MIMO over MIMO-LTE for MQAM considering Rayleigh fading distribution
}

\author{
Darshana Kaushik ${ }^{1}$, Ankita Rajkhowa ${ }^{2}$, Parismita Gogoi ${ }^{3}$, Bhargavjyoti Saikia ${ }^{4}$ \\ ${ }^{1}$ (Department of Electronics and Communication Engineering, Dibrugarh University Institute of Engineering \\ and Technology, Dibrugarh University, India) \\ ${ }_{2}^{2}$ (Department of Electronics and Communication Engineering, Dibrugarh University Institute of Engineering \\ and Technology, Dibrugarh University, India) \\ ${ }_{3}^{3}$ (Department of Electronics and Communication Engineering, Dibrugarh University Institute of Engineering \\ and Technology, Dibrugarh University, India) \\ ${ }^{4}$ (Department of Electronics and Communication Engineering, Dibrugarh University Institute of Engineering \\ and Technology, Dibrugarh University, India)
}

\begin{abstract}
LTE (Long Term Evolution) is a 3GPP (Third Generation Partnership Project) wireless standards which uses the standard OFDMA (Orthogonal Frequency Division Multiple Access) modulation, MU-MIMO (Multiuser Multiple Input Multiple Output) technology and different multipath fading models. LTE allows the operator to use spectrum more efficiently to deliver high speed data. This paper characterizes the downlink performance of LTE. The requirement for high data rate applications demanded a system to provide users with the MIMO technology which constitutes a breakthrough in wireless communication and is defined in the LTE standard. There are many metric to characterize the performance, but one of the most convenient and informative metric is the BER (Bit Error Rate). So the performance is characterized in terms of BER. In this paper the LTE system is modeled and simulated using MATLAB and the BER for $2 \times 2$ and $4 \times 4$ MIMO-LTE using 16QAM and 64QAM modulation schemes for Rayleigh fading environment are obtained against different SNR values.
\end{abstract}

Keywords: $3 G P P$, LTE, MIMO, PHY, BER, SNR

\section{Introduction}

Long Term Evolution (LTE) is a 4G wireless broadband technology developed by the Third Generation Partnership Project (3GPP). LTE has been designed to deliver users the benefit of the faster data speed as well as the high capacity voice support and new services by creating a new radio access technology that is optimized for IP based traffic. It will also provide network operators improvement in performance over the current high speed packet access system. LTE operates in the bands FDD (Frequency Division Duplex) and TDD (Time Division Duplex) of UMTS. LTE provides data rate of about 100Mbps in the downlink in $20 \mathrm{MHz}$ channel and $50 \mathrm{Mbps}$ in the uplink in $20 \mathrm{MHz}$ channel. The spectral efficiency of LTE is upto 4 times greater than HSPA (High Speed Packet Access). Scalable bandwidth of LTE is from 1.4 upto 20MHz. LTE make use of some advanced signal processing techniques so that the target can be achieved. These techniques are new to cellular application. LTE uses new technologies such as OFDMA (Orthogonal Frequency Division Multiple Access), which supports parallel transmission on large number of narrowband subcarriers with spacing of $50 \mathrm{KHz}$ is used in the downlink. This multiple access scheme protect against multipath and frequency selective fading and also for the simple equalization of the signal. It also improves the spectral efficiency. The uplink uses a scheme known as SCFDMA (Single Carrier Frequency Division Multiple Access). LTE uses multiple antenna techniques known as MIMO (Multiple Input Multiple Output). MIMO system often increases quality or capacity at no added spectrum cost. In the downlink different modes of MIMO are supported and there are various choices of MIMO configuration available. The modulation schemes used in LTE are QPSK, 16QAM, 64QAM for the downlink whereas in the uplink 64QAM is optional for the handset to support.

\section{Theoretical Background}

LTE was developed by the $3 \mathrm{GPP}$ and can be considered as an evolution of UMTS (Universal Mobile Telecommunication System) and HSPA (High Speed Packet Access). LTE began in 2004 and was completed in 2008 and was known as 3GPP Release 8. The goals of LTE are improved system capacity and coverage, high peak data rates, low latency, reduced operating costs, multi-antenna support, flexible bandwidth operations, seamless integration with existing systems (UMTS, Wi-Fi, etc.). The radio interface which covers the interface between the UE (User Equipment) and the network is composed of the Physical layer, Medium Access Control layer and the Radio Resource Control layer. The downlink air interface technologies used in LTE are- OFDMA and MIMO. In OFDMA technique multiple simultaneous users are allocated to different subcarriers. In the 
downlink per antenna port there is one resource grid. Depending on the reference signal configuration in the cell the set of antenna port is supported. The downlink resource grid consists of Resource block, Resource elements and Resource element-groups. A resource block consists of 12 subcarriers in the time domain and 7 OFDMA symbols in the frequency domain with a subcarrier spacing of $15 \mathrm{KHz}$ and several numbers of resource blocks combine together to form the resource grid. Thus in the time domain the entire band is divided into blocks of 12 subcarriers or $180 \mathrm{KHz}$. These blocks forms the so called Physical Resource Blocks (PRB). The subcarriers in these resource blocks are modulated using QPSK (Quadrature Phase Shift Keying), 16QAM (16 Quadrature Amplitude Modulation), 64QAM (64 Quadrature Amplitude Modulation) modulation techniques[1,2,3,4].

A message signal whose frequency spectrum does not fall within a fixed frequency range or is unsuitable for the channel, is transmitted by changing the carrier signal according to the information in the message signal. This alteration is called modulation, and it is the modulated signal that is transmitted. The receiver then recovers the original signal through a process called demodulation. Modulation schemes should achieve low bit error rate in the presence of fading, Doppler spread, interference and thermal noise. In 16QAM modulation, the constellation diagram have 16 different positions and each modulation symbol are mapped to different positions in the constellation diagram. Thus 16 QAM needs 4 bits to encode different modulation symbols. Similarly 64QAM have 64 different positions in the constellation diagram, so 6 bits are required to encode each modulation symbol. Higher order modulation i.e. 64QAM gives best result with strong signals and lower order modulation like QPSK gives best results with weak signals [5,6,7]. As specified in the LTE standard the preferred channel coding techniques for LTE are Turbo Coding and Rate Matching.

The requirement for high data rate applications demanded a system to provide users with a technology which constitutes a breakthrough in wireless communication. This technology led to the use of multiple number of transmitting and receiving antennas in wireless systems, known as MIMO (Multiple Input Multiple Output) technology which is defined in the LTE standard. In conventional wireless communications, a single antenna is used at the source, and another single antenna is used at the destination. In some cases, this gives rise to problems with multipath effects. When an electromagnetic field (EM field) is met with obstructions such as hills, canyons, buildings, and utility wires, the wave-fronts are scattered, and thus they take many paths to reach the destination. The late arrival of scattered portions of the signal causes problems such as fading. The use of two or more antennas, along with the transmission of multiple signals (one for each antenna) at the source and the destination, eliminates the trouble caused by multipath wave propagation, and can even take advantage of this effect. Thus MIMO wireless technology is able to considerably increase the capacity and throughput of a given channel by using multiple antennas. Improvement in capacity and BER (Bit Error Rate) is also seen by the use of MIMO. Thus MIMO can actually be said to be a radio antenna technology as it uses multiple antennas at the transmitter and the receiver to carry the data. The block diagram of a MIMO is shown in Figure 1. The channel with $\mathrm{N}$ outputs and $\mathrm{M}$ inputs is denoted as $\mathrm{M} \times \mathrm{N}$ matrix:[8,9,10]

$$
H(n)=\left(\begin{array}{ccc}
h_{(n, 1,1)} & \ldots & h_{(n, 1, N)} \\
\vdots & \ddots & \vdots \\
h_{(n, M, 1)} & \cdots & h_{(n, M, N)}
\end{array}\right)
$$

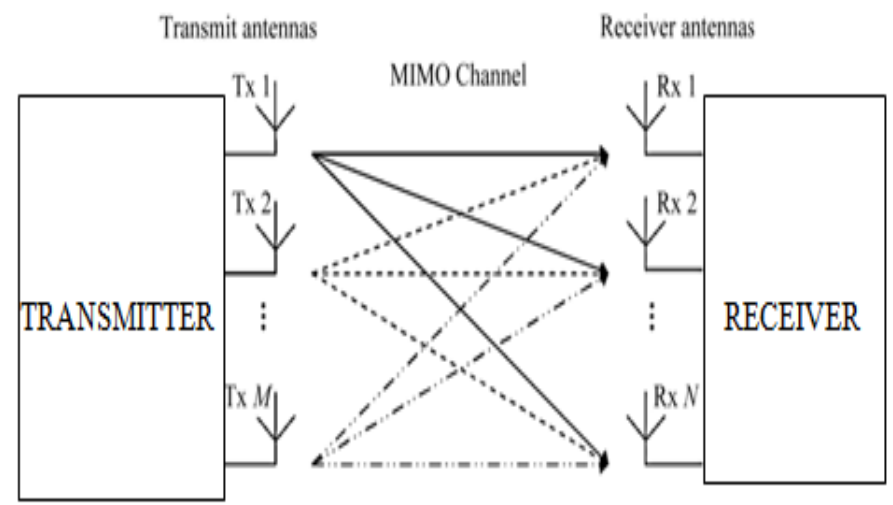

Fig. 1. MIMO (Multiple Input Multiple Output)

MIMO is a key component of next-generation wireless technologies and provides the bulk of LTE's peak throughput gains when compared with older technologies. However, MIMO gains can only be realized on a fully optimized network. MIMO optimization requires a different approach to traditional network optimization, with assessment of multipath conditions playing a key role in determining the potential throughput 
provided by a MIMO-enabled LTE network. Operators can use the knowledge gained from analyzing scanning receiver MIMO data to improve conditions in the current network through antenna and eNodeB adjustments. Scanning receiver data can also be used to evaluate the performance of UEs and eNodeB MIMO mode selection. Finally, accurate data on multipath conditions in existing LTE networks can lead to better planning of future MIMO-capable networks. This knowledge will become increasingly valuable as more users depend on LTE to provide the data rates they need for wireless applications from mobile banking to high-definition video streaming. Operators that maximize the performance of MIMO in their LTE networks will be able to provide the best service to these users with the smallest amount of infrastructure investment, providing a clear competitive advantage in both price and quality of service.[11]

In LTE standard four different types of MIMO algorithms are defined: receiver-combing, transmit diversity , beam-forming and spatial-multiplexing. [1]

In MRC the transmitted signal is estimated by averaging the multiple received signal. In SC the transmitted signal is estimated by using only the received signal with the highest SNR (Signal-to-Noise Ratio).

In transmit diversity scheme different antennas transmit the information at each sub-carrier. Data rate is not increased by this scheme but it only makes the communication link more robust. Transmit diversity uses space-time coding which is used to extract as much information as possible from the received signal by combining all the copies of the received signal in an optimal way. The transmit-diversity scheme used in LTE is STBC (Space-Time Block Coding ). Space-time block codes are used to improve the reliability of data transfer across a number of antennas[12,13,14]. STBC uses both spatial and temporal diversity. STBC involves transmission of multiple copies of data. This helps to compensate for the channel problems such as fading and thermal noise. Although there is redundancy in the data some copies may arrive less corrupted at the receiver. The matrix representation of STBC is shown below-

$\left(\begin{array}{ccc}S_{11} & \cdots & S_{1 n} \\ \vdots & \ddots & \vdots \\ s_{m 1} & \cdots & s_{m n}\end{array}\right)$

Each row represents a time slot and each column represents one antenna's transmissions over time. Within the above matrix, $S_{m n}$ is the modulated symbol to be transmitted in time slot ' $m$ ' from antenna ' $n$ '.

Spatial multiplexing increases the data rate due to its capability to transmit non-redundant information. It is susceptible to deficiencies in rank of the matrix representing the MIMO equation. Multiple techniques are introduced in LTE spatial multiplexing in order to minimize the probability of these rank deficiencies occurring and to harness its benefits. For $N_{t}$ transmit antennas and $N_{r}$ receive antennas, the maximum spatial multiplexing order is given by :-

$$
N_{s}=\min \left(N_{t}, N_{r}\right)
$$

here $N_{s}$ is the maximum spatial multiplexing order.

MIMO Flat Fading Channel Matrix at sample time $\mathrm{n}$ is given by:-

$$
H(n)=\left(\begin{array}{ccc}
h_{(n, 1,1)} & \cdots & h_{(n, 1, N)} \\
\vdots & \ddots & \vdots \\
h_{(n, M, 1)} & \cdots & h_{(n, M, N)}
\end{array}\right)
$$

Here the time index $n=1,2, \ldots \ldots . ., n$ Samp, where $n$ Samp is the number of transmitted symbols in each sub-frame per antenna and $M, N$ are the number of transmitted and received antennas respectively . The channel matrix of MIMO multipath fading channel is

$$
H(n, k)=\left(\begin{array}{ccc}
h_{(n, k, 1,1)} & \cdots & h_{(n, k, 1, N)} \\
\vdots & \ddots & \vdots \\
h_{(n, k, M, 1)} & \cdots & h_{(n, k, M, N)}
\end{array}\right)
$$

The path-delay index $\mathrm{k}$ is $k=1, \ldots \ldots, L$, where $\mathrm{L}$ is the number of path delays[15].

The most important challenge related to wider band transmission is the effect of multipath fading of the radio channel. Multipath fading is the result of the propagation of multiple versions of the transmitted signals through different paths before they arrive at the receiver. The different signals exhibit varying signal power and time delays or phases. As a result the received signal can be modeled as a filtered version of the transmitted signal that is filtered by the impulse response of the radio channel[1]. Fading can be classified into two types as fast fading and slow fading. The multipath propagation of the transmitted signal, which causes fast fading is because of the three propagation mechanisms described as reflection, diffraction and scattering. The multiple 
signal paths may sometimes add constructively or sometimes destructively at the receiver, causing a variation in the power level of the received signal. Rayleigh fading is the name given to the form of fading that is often experienced in an environment where there are a large number of reflections present. The Rayleigh fading model is normally viewed as a suitable approach to take when analysing and prediction radio wave propagation performance for areas such as cellular communications in a well built up urban environment where there are many reflections from buildings, etc. and a line of sight path is not there $[1,8,16,17]$.

In the 3GPP LTE standard three different multipath fading models are defined. They are EPA (Extended Pedestrian A), EVA (Extended Vehicular A), ETU (Extended Typical Urban). The delay profiles of these three models are shown in Table 1. In MIMO actually refers to sending and receiving multiple data on the same radio channel at the same time via multipath propagation and therefore there may be correlation between antenna port at both the transmitter and the receiver. It is always desirable to minimize this correlation[18]. In LTE standard three different correlation level are specified and they are shown in Table 2.[1]

Table 1. Delay profiles for E-UTRAN channel models

\begin{tabular}{|c|c|c|}
\hline Channel model & Excess tap delay (ns) & Relative power (dB) \\
\hline EPA & 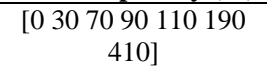 & $\begin{array}{c}0-1-2-3-8-17.2- \\
20.8]\end{array}$ \\
\hline EVA & 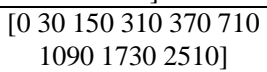 & $\begin{array}{c}{[0-1.5-1.4-3.6-0.6-} \\
9.1-7-12-16.9]\end{array}$ \\
\hline ETU & 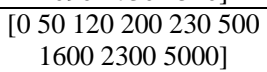 & {$\left[\begin{array}{lllllllll}-1 & -1 & -1 & 0 & 0 & 0 & -3 & -5 & -7\end{array}\right]$} \\
\hline
\end{tabular}

Table 2. Correlation matrices for high, medium and low correlation

\begin{tabular}{|c|c|c|}
\hline $\begin{array}{c}\text { LTE MIMO channel } \\
\text { correlation levels }\end{array}$ & $\boldsymbol{\alpha}$ & $\boldsymbol{\beta}$ \\
\hline Low correlation & 0 & 0 \\
\hline Medium correlation & 0.3 & 0.9 \\
\hline High correlation & 0.9 & 0.9 \\
\hline
\end{tabular}

In reference [19], the BER vs. SNR performance for an LTE MIMO system is observed for a Rayleigh fading environment using different modulation schemes like QPSK and 16QAM. In reference [20], the BER vs. SNR for $2 \times 2$ and $4 \times 4$ MIMO-LTE using QPSK modulation schemes and MMSE equalizer is plotted and the BER values for Rayleigh fading channel are compared for 2x2 MIMO-LTE and 4x4 MIMO-LTE for the modulation schemes. But in this paper focus is mainly on the downlink LTE in Rayleigh fading environments for $2 \times 2$ and $4 \times 4$ MIMO-LTE using 16QAM and 64QAM modulation schemes. So for the transmission of the data a better understanding of the LTE PHY radio interface is important.

\section{System Model}

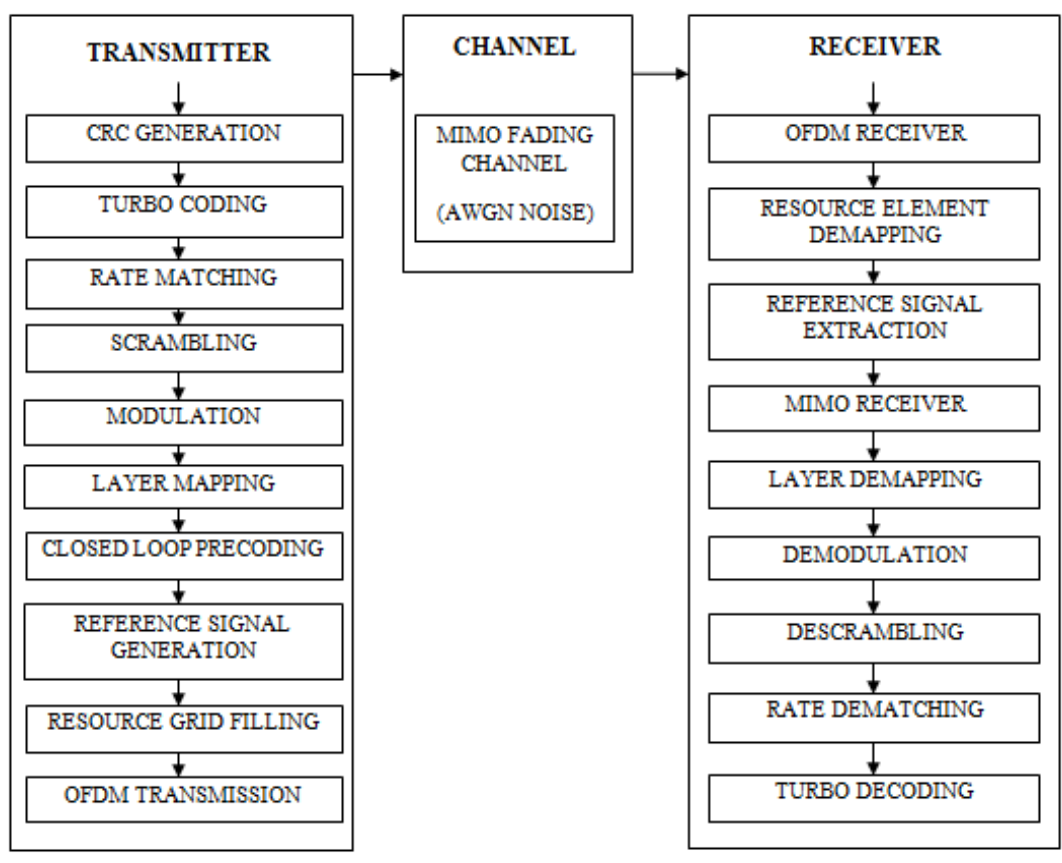

Fig. 2. System Model of LTE Downlink 
The structure of the LTE downlink transceiver is shown in Figure 2 . It is composed of a transmitter, a channel model and a receiver. The LTE Physical layer which is interfaced by the MAC layer is connected by the transport channels. In the transmitter side the transport channel performs CRC (Cyclic Redundancy Code) generation, turbo coding based on $1 / 3$ rate and rate matching of the coded transport channel to handle any requested coding rates. The coded bits are then scrambled resulting in a block of scrambled bits, according to:

$\tilde{b}^{(q)}(i)=\left(b^{(q)}(i)+c^{(q)}(i)\right) \bmod 2$

where $c^{(q)}(i)$ is the block of scrambling sequence based on codeword $q$ and $b^{(q)}(i)$ is the block of coded bits based on the codeword q. The blocks of scrambled bits are then modulated using modulation mapper, which produces complex valued modulation symbols:

$x=I+j Q$

where I is the In-phase component and Q is the Quadrature components of the modulated symbol. The complex valued modulated symbols are mapped onto one or several layers :

$x(i)=\left[x^{(0)}(i) \ldots \ldots \ldots \ldots . . . . x^{(v-1)}(i)\right]^{T}$

where $i=0,1,2, \ldots \ldots . . . . M_{\text {symb }}^{\text {layer }}-1,0$ is the number of layers and $M_{\text {symb }}^{\text {layer }}$ is the number of modulation symbols per layer. The block of vectors from the layered mapping is then precoded using the precoder and produces an output:

$$
\begin{aligned}
& y(i)=\left[\ldots \ldots \ldots . . y^{p}(i) \ldots \ldots \ldots \ldots\right]^{T} \\
& \text { where } i=0,1,2, \ldots \ldots \ldots . M_{\text {symb }}^{a p}-1 \text { and } y^{p}(i) \text { represents the signal for each antenna port } p .
\end{aligned}
$$

To properly decode the data the terminal in LTE needs Reference Signals. After Precoding reference signals are generated, the coded bits are then mapped to the physical resource blocks. Then OFDM (Orthogonal Frequency Division Multiplexing) transmission is applied to the resource grid which produces the transmitted symbols. The transmitted symbols are then passed through a MIMO fading channel which is distorted by AWGN noise. After the symbols are passed through the channel it will be received at the receiver and in the receiver the symbols will be recovered based on the reverse operations as on the transmitter side. The receiver will receive the OFDM signals processed by the channel, an estimate of the noise variance per received channel, the transmitted reference signals and demodulate the OFDM symbols to generate the best estimate of the transmitted symbols.[14,19,20]

\section{Experimental Results}

The information bits are generated in the transmitter side. The bits are than modulated using different modulation schemes such as 16 QAM and 64 QAM. The modulated bits are transmitted using two transmitting antennas and sent to the receiver through MIMO Fading Channel and AWGN noise is added. The transmitted signal is received by two receiving antennas at the front end of the receiver. It is then demodulated to get the desired signal. Finally the signal is sent to the user. Bit-error rate (BER) is plotted against different SNR (Signal to Noise ratio) values. The system has been developed using communication tool box available in MATLAB.

\subsection{PARAMETERS USED}

The parameters used are listed in Table 3, Table 4 and Table 5.

Table 3. Cell Width Configuration

\begin{tabular}{|c|c|}
\hline PARAMETERS & VALUES \\
\hline No of Frames & 1024 \\
\hline No of resource blocks & 50 \\
\hline No of Transmit antennas & $2 / 4$ \\
\hline Cell ID & 0 \\
\hline Cyclic prefix & Normal (7) \\
\hline Duplex mode & FDD (Frequency Division Duplex) \\
\hline & $8: 13(16 \mathrm{QAM} 2 \times 2)$ \\
SNR Range & $8: 16(16 \mathrm{QAM} 4 \times 4)$ \\
& $16: 24(64 \mathrm{QAM} 2 \times 2)$ \\
& $16: 24(64 \mathrm{QAM} 4 \times 4)$ \\
\hline
\end{tabular}


Table 4. Channel Model Configuration

\begin{tabular}{|c|c|}
\hline PARAMETERS & VALUES \\
\hline No of received antennas & $2 / 4$ \\
\hline Downlink channel & PDSCH (Physical downlink shared channel) \\
\hline Channel bandwidth & $20 \mathrm{MHz}$ \\
\hline Channel model & EVA 5Hz \\
\hline Doppler shift & 5 \\
\hline Fading type & Rayleigh \\
\hline Noise type & AWGN \\
\hline MIMO correlation & Low \\
\hline Normalize path gain & On \\
\hline Normalize for transmission antennas & On \\
\hline
\end{tabular}

Table 5. Modulation and Equalization

\begin{tabular}{|c|c|}
\hline PARAMETERS & VALUES \\
\hline Transmission mode & 4 (Spatial multiplexing) \\
\hline Modulation & 16QAM, 64QAM \\
\hline Mode type & 16QAM, 64QAM \\
\hline Coding rate & 1/3 \\
\hline Equalization mode & MMSE \\
\hline Demodulation & 16QAM, 64QAM \\
\hline
\end{tabular}

\subsection{BER vs. SNR PLOTS}

The BER is defined as the quality of the digital link which is calculated from the number of bits received with error divided by the number of bits transmitted by the transmitter and SNR is defined as the ratio of the received signal strength over the noise strength in the frequency range of the operation. SNR is inversely related to BER, that is high BER causes low SNR. High BER causes increase in delay and decreases throughput. SNR is used to study the quality of a communication link.

The BER vs. SNR plots for 16QAM $2 \times 2$ and $4 \times 4$ MIMO-LTE using LTE PHY transceiver model for Rayleigh fading environment are shown in Figure 3 and Figure 4 respectively. BER values for $2 \times 2$ and $4 \times 4$ MIMO-LTE for different values of SNR for16QAM Rayleigh fading are listed in Table 6 and Table 7 respectively.

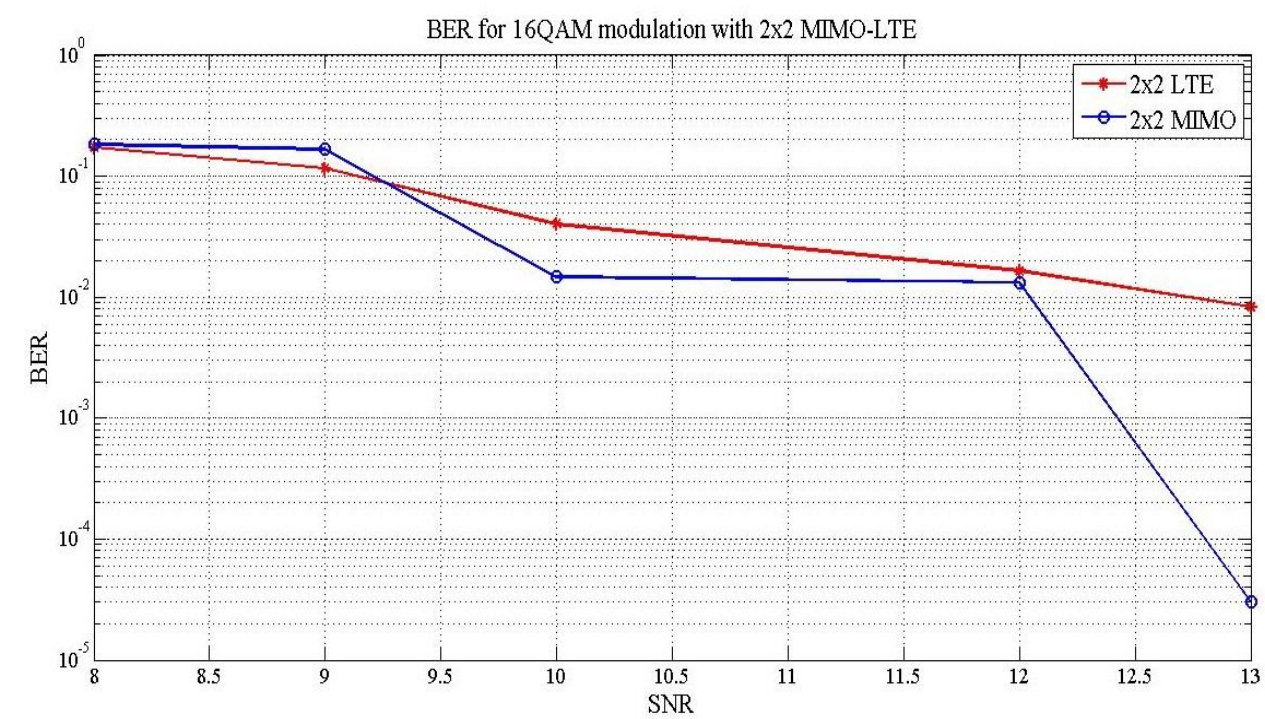

Fig. 3. BER vs. SNR plot for $2 \times 2$ MIMO-LTE with 16QAM modulation for RAYLEIGH fading

Table 6. BER vs. SNR values for $2 \times 2$ MIMO-LTE with 16QAM modulation for RAYLEIGH fading

\begin{tabular}{|c|c|c|c|}
\hline \multirow{2}{*}{ Sl. No } & \multirow{2}{*}{ SNR $(\mathbf{d b})$} & \multicolumn{2}{|c|}{ BER 16QAM 2x2 MIMO-LTE } \\
\cline { 3 - 4 } & 8 & MIMO & LTE \\
\hline 1 & 9 & 0.1822 & 0.1731 \\
\hline 2 & 10 & 0.166 & 0.1163 \\
\hline 3 & 12 & 0.01465 & 0.04025 \\
\hline 4 & 13 & 0.01318 & 0.01651 \\
\hline 5 & $2.998 \times \times^{10^{-5}}$ & 0.008272 \\
\hline
\end{tabular}




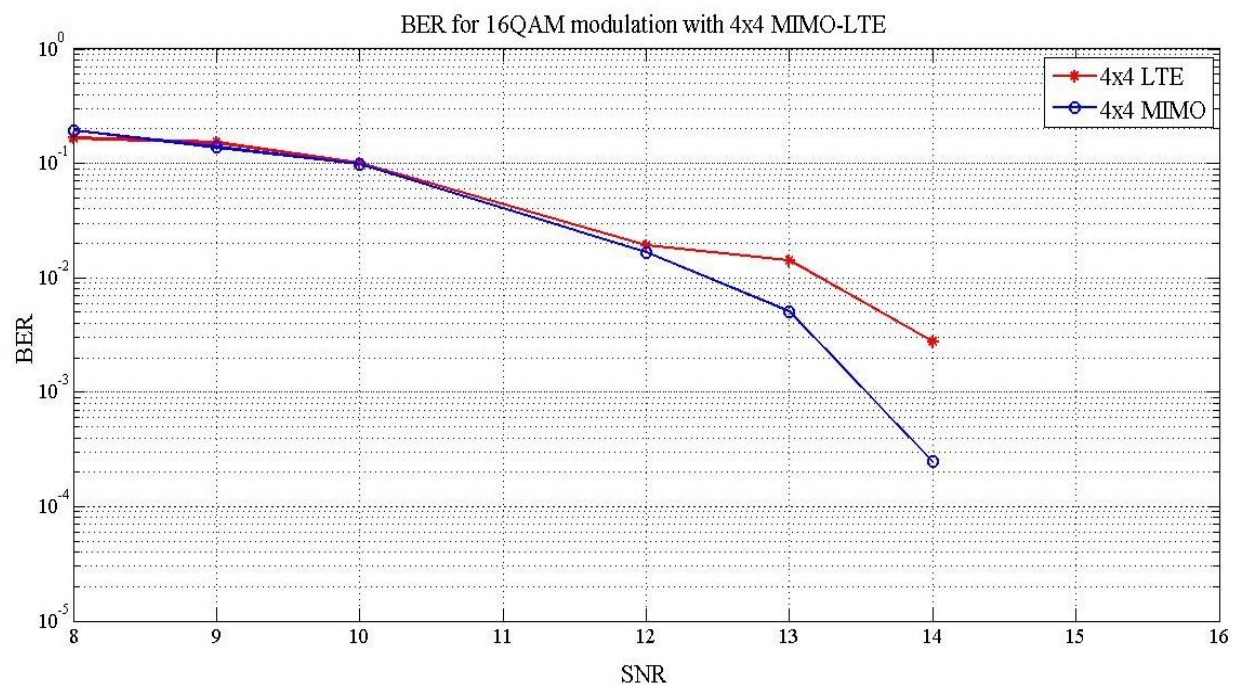

Fig. 4. BER vs. SNR plot for $4 \times 4$ MIMO-LTE with 16QAM modulation for RAYLEIGH fading

Table 7. BER vs. SNR values for $4 \times 4$ MIMO-LTE with 16QAM modulation for RAYLEIGH fading

\begin{tabular}{|c|c|c|c|}
\hline \multirow{2}{*}{ Sl. No } & \multirow{2}{*}{ SNR $(\mathbf{d b})$} & \multicolumn{2}{|c|}{ BER 16QAM 4x4 MIMO-LTE } \\
\cline { 2 - 4 } & 8 & MIMO & LTE \\
\hline 1 & 9 & 0.1942 & 0.1669 \\
\hline 2 & 10 & 0.1383 & 0.1523 \\
\hline 3 & 12 & 0.0988 & 0.1012 \\
\hline 4 & 13 & 0.01678 & 0.01918 \\
\hline 5 & 14 & 0.005097 & 0.0142 \\
\hline 6 & & 0.0002462 & 0.002774 \\
\hline
\end{tabular}

Also the BER vs. SNR plots for 64QAM $2 \times 2$ and $4 \times 4$ MIMO-LTE using LTE PHY transceiver model for Rayleigh fading environment are shown in Figure 5 and Figure 6 respectively. BER values for $2 \times 2$ and $4 \times 4$ MIMO-LTE for different values of SNR for 64QAM Rayleigh fading are listed in Table 8 and Table 9 respectively.

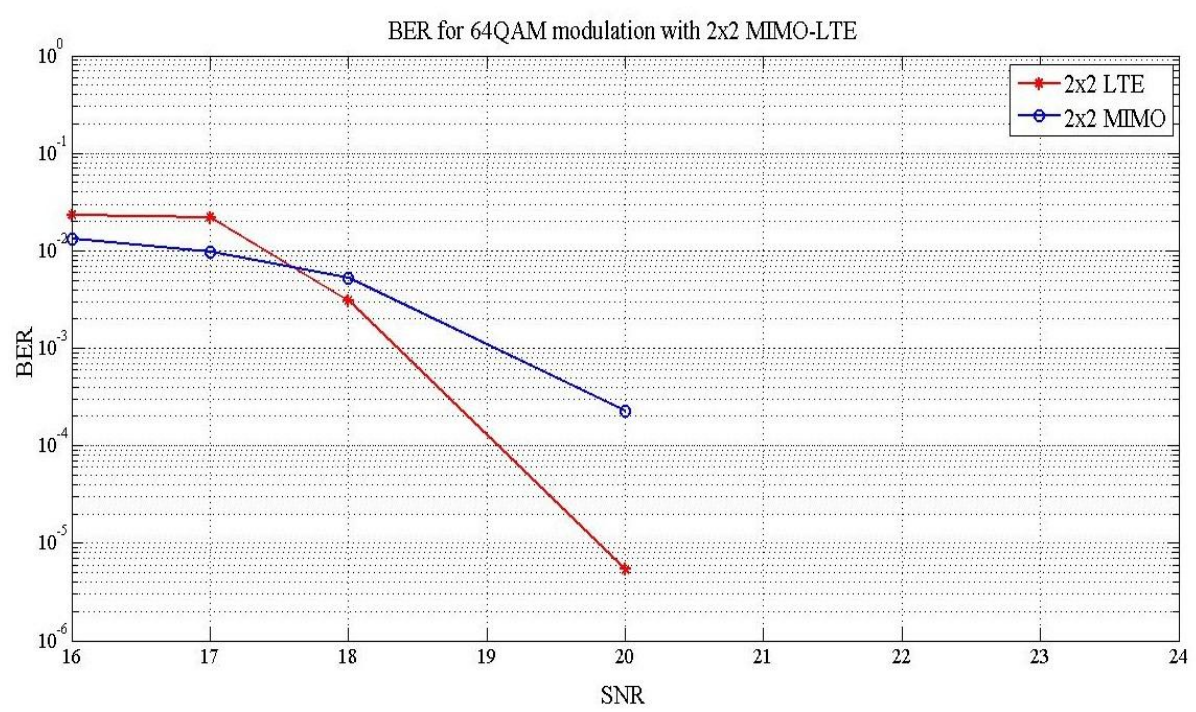

Fig. 5 BER vs. SNR plot for 2×2 MIMO-LTE with 64QAM modulation for RAYLEIGH fading

Table 8. BER vs. SNR values for $2 \times 2$ MIMO-LTE with 64QAM modulation for RAYLEIGH fading

\begin{tabular}{|c|c|c|c|}
\hline \multirow{2}{*}{ SI. No } & \multirow{2}{*}{ SNR $(\mathbf{d b})$} & \multicolumn{2}{|c|}{ BER 64QAM 2x2 MIMO-LTE } \\
\cline { 3 - 4 } & 16 & MIMO & LTE \\
\hline 1 & 17 & 0.01322 & 0.02314 \\
\hline 2 & 18 & 0.009731 & 0.02183 \\
\hline 3 & 20 & 0.005245 & 0.003068 \\
\hline 4 & & 0.0002273 & $5.389 \times \times^{10^{-8}}$ \\
\hline
\end{tabular}




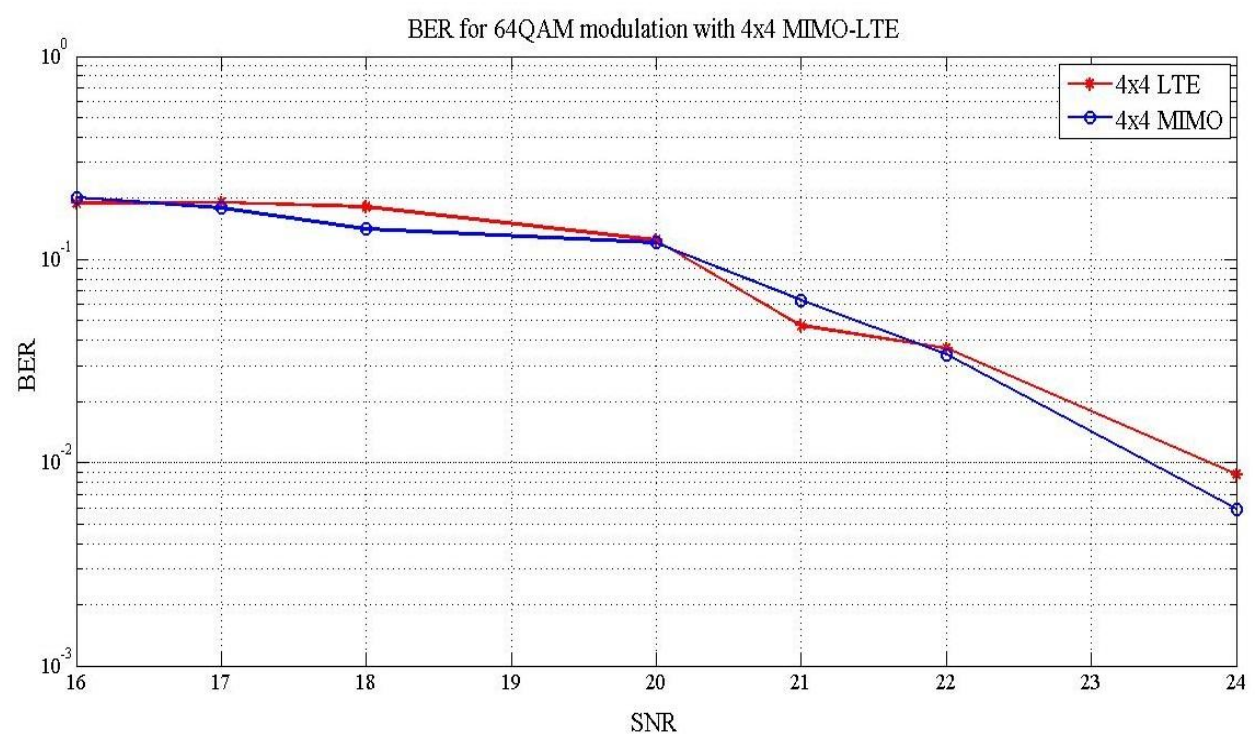

Fig. 6. BER vs. SNR plot for $4 \times 4$ MIMO-LTE with 64QAM modulation for RAYLEIGH fading

Table 9. BER vs. SNR values for 4×4 MIMO-LTE with 64QAM modulation for RAYLEIGH fading

\begin{tabular}{|c|c|c|c|}
\hline \multirow{2}{*}{ Sl. No } & \multirow{2}{*}{ SNR $(\mathbf{d b})$} & \multicolumn{2}{|c|}{ BER 64QAM 4x4 MIMO-LTE } \\
\cline { 3 - 4 } & 16 & MIMO & LTE \\
\hline 1 & 17 & 0.2023 & 0.1892 \\
\hline 2 & 18 & 0.1788 & 0.1914 \\
\hline 3 & 20 & 0.1412 & 0.1818 \\
\hline 4 & 21 & 0.1208 & 0.1247 \\
\hline 5 & 22 & 0.06298 & 0.04721 \\
\hline 6 & 24 & 0.03398 & 0.03641 \\
\hline 7 & & 0.005893 & 0.008744 \\
\hline
\end{tabular}

\section{Conclusion}

3GPP LTE which uses MIMO seems to be cornerstone due to the potential increase in data rate and transmit diversity offered by MIMO technology. In this paper the concept of 3GPP LTE has been demonstrated using the standard LTE model with transmission mode 4 with severely faded EVA $5 \mathrm{~Hz}$ fading channel. The signal is transmitted through Rayleigh faded environment. BER curves play a very important role in the performance of the system. With the increasing value of SNR, the BER values must decrease for a better performance of the system. In this paper the BER vs. SNR for $2 \times 2$ and $4 \times 4$ MIMO-LTE using 16QAM and 64 QAM modulation schemes and MMSE equalizer is plotted and the BER values for Rayleigh fading channel are compared for $2 \times 2$ and $4 \times 4$ MIMO-LTE are compared for the modulation schemes. From the analysis this can be conclude that the BER reduce in LTE-MIMO significantly when the SNR is increased. But with increase of antenna nos. for low SNR, due to the antenna correlation, a random BER performance can be seen.

Further the work can be extended using a better equalizer to get a better link performance between the transmitter and the receiver. In this paper although EVA $5 \mathrm{~Hz}$ fading channel is considered, several other severely faded channels can be used. The no of transmit and receive antennas can also be increased.

\section{References}

[1] Dr Houman Zarrinkoub, Understanding LTE with Matlab® From Mathematical Modeling to Simulation and Prototyping Wiley, 2014.

[2] C. Gessner, A. Roessler, M. Kottkamp, UMTS Long Term Evolution (LTE) - Technology Introduction, LTE Technology Introduction Rohde \& Schwarz, July 2012, 1MA111_3E.

[3] S. Sesia, I Toufik, M. Baker, LTE - The UMTS Long Term Evolution From Theory to Practice @ 2009 John Wiley \& Sons Ltd.

[4] T. Nakamura, Proposal for Candidate Radio Interface Technologies for IMT -Advanced Based on LTE Release 10 and Beyond (LTE -Advanced) (C) 3GPP 2009 Mobile World Congress, Barcelona, 19 @ 3GPP 2009

[5] B. Sklar, Digital Communication Prentice Hall, Upper Saddle River, NJ, Second Edition, 2008.

[6] S. Haykin, Communications Systems Second Edition, Wiley, 1983.

[7] J. Proakis, Digital Communication 5th Edition, McGraw-Hill, 2007.

[8] A. Goldsmith, Wireless Communication Cambridge University Press, 2005.

[9] T.S. Rappaport, Wireless Communications-Principles and Practice Pearson Education 1997.

[10] R. U. Nabar A. J. Paulraj, D. A. Gore and H. B“"olcskei, An overview of MIMO communications-- a key to gigabit wireless Proceedings of the IEEE, vol. 92, no. 2, pp. 198-218, Feb. 2004.

[11] Brian Stetler, Maximizing LTE Performance Through MIMO Optimization White paper, Gadgetwise (blog), New York Times, January 7, 2011.

[12] C. Cox, An Introduction to LTE, LTE-advanced, SAE, VoLTE and 4G mobile communications Second Edition, Wiley, 2014. 
[13] Juho. Lee, Jin-Kyu Han and Jianzhong (Charlie) Zhang, Review Article MIMO Technologies in 3GPP LTE and LTE-Advanced Hindwai Publishing Corporation, EURASIP Journal on Wireless Communications and Networking, Volume 2009.

[14] D. Gesbert,M. Shafi, D. shan Shiu, P. J. Smith, and A. Naguib, From theory to practice: an overview ofMIMO space-time coded wireless systems IEEE J. Selected Areas Comm., vol. 21, pp. 281-302, 2003.

[15] P. Gogoi, R. Borah, A. Sarma, B. Saikia, On The Evolution of Downlink Physical Layer in multi-antenna 3GPP LTE / LTE-A: A review 2015 International Symposium on Advanced Computing and Communication.

[16] M. K. Simon, M.-Slim Alouini, Digital Communication over Fading Channels 2nd ed. New York: John Wiley and Sons, 2005.

[17] G. J. Foschini and M. J. Gans, On limits of wireless communications in a fading environment when using multiple antennas WirelessPersonal Communications, vol. 6, pp. 311-335, Feb. 1998.

[18] A. F. Molisch and F. Tufvesson, Multipath propagation models for broadband wireless systems in CRC Handbook of signal processing for wireless commmunications (M. Ibnkahla, ed.), p. in press, 2003.

[19] D. Kaushik, R. Borah, P. Gogoi, 3GPP LTE Downlink PHY transceiver using Closed-loop Spatial Multiplexing in Frequency Selective Fading Environment International Journal of Computer Applications (0975 - 8887) Volume 134 - No.12, January 2016.

[20] A. Rajkhowa, D. Kaushik, B.J. Saikia, P. Gogoi, Performance Analysis of MIMO over MIMO-LTE for QPSK considering Rayleigh Fading Distribution International Journal of Research and Scientific Innovation (IJRSI) | Volume III, Issue VI, June 2016 | ISSN 23212705 . 